International Journal of Pure and Applied Mathematics

Volume 87 No. 4 2013, 529-534

ISSN: 1311-8080 (printed version); ISSN: 1314-3395 (on-line version)

url: http://www.ijpam.eu

doi: http://dx.doi.org/10.12732/ijpam.v87i4.3

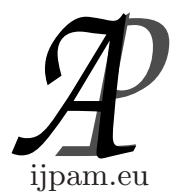

\title{
AN EXTENSION OF POISSON APPROXIMATION BY $w$-FUNCTIONS
}

\author{
K. Teerapabolarn \\ Department of Mathematics \\ Faculty of Science \\ Burapha University \\ Chonburi, 20131, THAILAND
}

\begin{abstract}
We extend the result of Poisson approximation [4] to approximate the distribution of a sum of independent non-negative integer-valued random variables. A general upper bound for the total variation distance in terms of each $w$-function is obtained. Examples have been given to illustrate applications of the result.
\end{abstract}

AMS Subject Classification: 62E17, 60F05, 60G50

Key Words: Poisson approximation, Stein-Chen method, $w$-functions

\section{Introduction}

Let $X_{1}, \ldots, X_{n}$ be $n$ independent non-negative integer-valued random variables, each with distribution $\mathcal{L}\left(X_{i}\right)$, mean $\mu_{i}$ and variance $\sigma_{i}^{2}$. Let $X=\sum_{i=1}^{n} X_{i}$ and $\mathcal{L}(X)$ and $\mathcal{P}(\lambda)$ denote the distribution of $X$ and the Poisson distribution with mean $\lambda$, respectively. It is well known that $\mathcal{L}(X)$ can be approximated by $\mathcal{P}(\lambda)$ provided natural conditions concerning their parameters are satisfied. For example, if $X$ is a sum of $n$ independent Bernoulli random variables with

Received: May 7, 2013

(c) 2013 Academic Publications, Ltd. url: www.acadpubl.eu 
each probability of success $p_{i}>0$ and all $p_{i}$ are small, then $\mathcal{L}(X)$ can be approximated by $\mathcal{P}(\lambda)$ with mean $\lambda=\sum_{i=1}^{n} p_{i}$.

In general, the accuracy of the approximation is usually measured in terms of the the total variation distance between two distributions and its upper bound. The total variation distance between $\mathcal{L}(X)$ and $\mathcal{P}(\lambda)$ is denoted and defined as

$$
d_{T V}(\mathcal{L}(X), \mathcal{P}(\lambda))=\sup _{A}|\mathcal{L}(X)(A)-\mathcal{P}(\lambda)(A)|,
$$

where $A$ is a subset of $\mathbb{N} \cup\{0\}$.

Many methods have been developed and applied to deal with the Poisson approximation, e.g., an interesting alternative method in Majsnerowska [4]. He used the idea of [3] to give an upper bound for approximating the distribution of a non-negative integer-valued random variable by the Poisson distribution with mean $\lambda$ in the form of

$$
d_{T V}(\mathcal{L}(X), \mathcal{P}(\lambda)) \leq \frac{1-e^{-\lambda}}{\lambda} \mathbb{E}\left|\lambda-\sigma^{2} w(X)\right|+\min \left\{1, \lambda^{-1 / 2}\right\}|\lambda-\mu|,
$$

where $X$ is a non-negative integer-valued random variable having a distribution $\mathcal{L}(X)$, mean $\mu$ and variance $\sigma^{2}, w(X)$ is the $w$-function associated with the random variable $X$. Observe that the result (1.2) was formulated for approximating the distribution of a random variable $X$. In the case where $X$ is a sum $\sum_{i=1}^{n} X_{i}$ of $n$ independent non-negative integer-valued random variables, it may not be applied. In this paper, we give an extension of the result (1.2) for approximating the distribution of such summands by the Poisson distribution.

The tools for giving the result consist of the Stein-Chen method and $w$ functions, which are in Section 2. In Section 3, we determine a bound for the total variation distance between $\mathcal{L}(X)$ and $\mathcal{P}(\lambda)$. For applications, we give some examples to illustrate the obtained result, which are in the last section.

\section{Method}

We use the same methodology as in [4], which consists of the Stein-Chen method and $w$-functions as follows.

For $w$-functions, let $p_{X}(x)$ and $\mathcal{S}(x)$ be the probability function and the support of a non-negative integer-valued random variable $X,[4]$ expressed a function $w$ associated with $X$ in the form of 


$$
\begin{aligned}
w(0)=\frac{\mu}{\sigma^{2}}, \quad w(x)=\frac{1}{\sigma^{2}}\left\{\mu+\frac{\sigma^{2} w(x-1) p_{X}(x-1)}{p_{X}(x)}-\right. & x\}, \\
x & \in \mathcal{S}(x) \backslash\{0\},
\end{aligned}
$$

where $w(x) \geq 0$ and $p_{X}(x)>0$ for every $x \in \mathcal{S}(x)$. The following relation is an important property for proving the result, which was stated by [2].

If a non-negative integer-valued random variable $X$ is defined as in Section 1 , then

$$
\operatorname{Cov}(X, f(X))=\sigma^{2} \mathbb{E}[w(X) \Delta f(X)],
$$

for any function $f: \mathbb{N} \cup\{0\} \rightarrow \mathbb{R}$ for which $\mathbb{E}|w(X) \Delta f(X)|<\infty$, where $\Delta f(x)=f(x+1)-f(x)$. We note that $\mathbb{E}[w(X)]=1$.

For the Stein-Chen method, it can be applied Stein's equation of the Poisson distribution with mean $\lambda>0$ for every subset $A$ of $\mathbb{N} \cup\{0\}$ and the bounded real valued function $f=f_{A}: \mathbb{N} \cup\{0\} \rightarrow \mathbb{R}$, defined as in [1], yields

$$
\mathcal{L}(X)\{A\}-\mathcal{P}(\lambda)\{A\}=\mathbb{E}[\lambda f(X+1)-X f(X)]
$$

For any subset $A$ of $\mathbb{N} \cup\{0\}$ and $k \in \mathbb{N} \cup\{0\},[1]$ showed that

$$
\sup _{k}|\Delta f(k)|=\sup _{k}|f(k+1)-f(k)| \leq \lambda^{-1}\left(1-e^{-\lambda}\right)
$$

and

$$
\sup _{k}|f(k)| \leq \sqrt{\frac{2}{\lambda e}}
$$

where $f(0)=0$.

\section{Result}

The following theorem presents the desired result of this study.

Theorem 3.1. Let $X=\sum_{i=1}^{n} X_{i}$, each with corresponding $w$-function $w_{i}\left(X_{i}\right)$, and let $\lambda=\sum_{i=1}^{n} \lambda_{i}, \mu=\sum_{i=1}^{n} \mu_{i}$ and $\sigma^{2}=\sum_{i=1}^{n} \sigma_{i}^{2}$. Then the following inequality hold:

$$
d_{T V}(\mathcal{L}(X), \mathcal{P}(\lambda)) \leq \frac{1-e^{-\lambda}}{\lambda} \sum_{i=1}^{n} \mathbb{E}\left|\lambda_{i}-\sigma_{i}^{2} w_{i}\left(X_{i}\right)\right|
$$




$$
+\sqrt{\frac{2}{\lambda e}}|\lambda-\mu|\left[1-p_{X}(0)\right]
$$

and if $\lambda=\mu$, then

$$
d_{T V}(\mathcal{L}(X), \mathcal{P}(\lambda)) \leq \frac{1-e^{-\lambda}}{\lambda} \sum_{i=1}^{n} \mathbb{E}\left|\lambda_{i}-\sigma_{i}^{2} w_{i}\left(X_{i}\right)\right|
$$

Proof. We shall show that (3.1) holds. From (2.3), it follows that

$$
\begin{aligned}
d_{T V}(\mathcal{L}(X), \mathcal{P}(\lambda)) & =|\lambda \mathbb{E}[f(X+1)]-\mathbb{E}[X f(X)]| \\
& =|\lambda \mathbb{E}[f(X+1)]-\mathbb{C o v}(X, f(X))-\mu \mathbb{E}[f(X)]| \\
& =\left|\sum_{i=1}^{n} \lambda_{i} \mathbb{E}[\Delta f(X)]-\sum_{i=1}^{n} \operatorname{Cov}\left(X_{i}, f(X)\right)+(\lambda-\mu) \mathbb{E}[f(X)]\right| .
\end{aligned}
$$

By (2.2) and for each $i$,

$$
\begin{aligned}
\mathbb{C o v}\left(X_{i}, f(X)\right) & =\mathbb{E}\left[\mathbb{C o v}\left(X_{i}, f\left(X_{i}+\sum_{j \neq i}^{n} X_{j}\right) \mid \sum_{j \neq i}^{n} X_{j}\right\rfloor\right. \\
& =\mathbb{E}\left\{\sigma_{i}^{2} \mathbb{E}\left[w_{i}\left(X_{i}\right) \Delta f\left(X_{i}+\sum_{j \neq i}^{n} X_{j} \mid \sum_{j \neq i}^{n} X_{j}\right]\right\}\right. \\
& =\sigma_{i}^{2} \mathbb{E}\left[w_{i}\left(X_{i}\right) \Delta f(X)\right],
\end{aligned}
$$

thus, by (2.4),

$$
\begin{aligned}
d_{T V}(\mathcal{L}(X), \mathcal{P}(\lambda)) & \leq \sum_{i=1}^{n} \mathbb{E}\left\{\left|\lambda_{i}-\sigma_{i}^{2} w_{i}\left(X_{i}\right)\right||\Delta f(X)|\right\}+|\lambda-\mu| \mathbb{E}|f(X)| \\
& \leq \frac{1-e^{-\lambda}}{\lambda} \sum_{i=1}^{n} \mathbb{E}\left|\lambda_{i}-\sigma_{i}^{2} w_{i}\left(X_{i}\right)\right|+|\lambda-\mu| \mathbb{E}|f(X)| .
\end{aligned}
$$

Since $f(0)=0$ and using $(2.5)$, we have

$$
E|f(X)|=\sum_{k \geq 1}|f(k)| p_{X}(k) \leq \sqrt{\frac{2}{\lambda e}} \sum_{k \geq 1} p_{X}(k)=\sqrt{\frac{2}{\lambda e}}\left[1-p_{X}(0)\right] .
$$

Hence, by (3.3) and (3.4), (3.1) holds. 
The following Corollary is a consequence of (3.1) under some conditions.

Corollary 3.1. For $i \in\{1, \ldots, n\}$, if $\lambda_{i}-\sigma_{i}^{2} w_{i}\left(x_{i}\right) \geq /<0$ for every $x_{i} \in \mathcal{S}\left(x_{i}\right)$, then

$$
d_{T V}(\mathcal{L}(X), \mathcal{P}(\lambda)) \leq\left(1-e^{-\lambda}\right)\left|1-\frac{\sigma^{2}}{\lambda}\right|+\sqrt{\frac{2}{\lambda e}}|\lambda-\mu|\left[1-p_{X}(0)\right]
$$

and if $\lambda=\mu$, then

$$
d_{T V}(\mathcal{L}(X), \mathcal{P}(\lambda)) \leq\left(1-e^{-\lambda}\right)\left|1-\frac{\sigma^{2}}{\lambda}\right|
$$

\section{Applications}

We give some examples to illustrate applications of Theorem 3.1 and Corollary 3.1 .

Example 4.1. Let $X_{1}, \ldots, X_{n}$ be $n$ independent Bernoulli random variables, each with probability function $p_{X_{i}}(k)=p_{i}^{k}\left(1-p_{i}\right)^{1-k}, k=0,1$. Then $\mu_{i}=p_{i}$, $\sigma_{i}^{2}=p_{i}\left(1-p_{i}\right)$ and $w_{i}(k)=\frac{(1-k) p_{i}}{\sigma_{i}^{2}}$. Setting $\lambda_{i}=\mu_{i}$ for every $1 \leq i \leq n$ in Theorem 3.1, it follows that $\lambda_{i}-\sigma_{i}^{2} w_{i}(k)=k p_{i} \geq 0$ for $k=0,1$. Therefore, by Corollary 3.1, we obtain

$$
d_{T V}(\mathcal{L}(X), \mathcal{P}(\lambda)) \leq \frac{1-e^{-\lambda}}{\lambda} \sum_{i=1}^{n} p_{i}^{2},
$$

where $\lambda=\sum_{i=1}^{n} p_{i}$. This is precisely the best known result in [1].

Example 4.2. Let $X_{1}, \ldots, X_{n}$ be $n$ independent geometric random variables, each with the probability function $p_{X_{i}}(k)=p_{i}\left(1-p_{i}\right)^{k}, k=0,1, \ldots$ Then $\mu_{i}=\frac{1-p_{i}}{p_{i}}, \sigma_{i}^{2}=\frac{1-p_{i}}{p_{i}^{2}}$ and $w_{i}(k)=\frac{(1+k)\left(1-p_{i}\right)}{p_{i} \sigma_{i}^{2}}$. Setting $\lambda_{i}=\mu_{i}$ for every $1 \leq i \leq n$ in Theorem 3.1, it follows that $\lambda_{i}-\sigma_{i}^{2} w_{i}(k)=-\frac{k\left(1-p_{i}\right)}{p_{i}} \leq 0$ for $k=0,1, \ldots$. Therefore, by Corollary 3.1 , we obtain

$$
d_{T V}(\mathcal{L}(X), \mathcal{P}(\lambda)) \leq \frac{1-e^{-\lambda}}{\lambda} \sum_{i=1}^{n} \frac{\left(1-p_{i}\right)^{2}}{p_{i}^{2}},
$$

where $\lambda=\sum_{i=1}^{n} \frac{1-p_{i}}{p_{i}}$. 


\section{References}

[1] A.D. Barbour, L. Holst, S. Janson, Poisson Approximation, Oxford Studies in Probability 2, Clarendon Press, Oxford, 1992.

[2] T. Cacoullos, V. Papathanasiou, Characterization of distributions by variance bounds, Statist. Probab. Lett., 7 (1989), 351-356.

[3] T. Cacoullos, V. Papathanasion, S.A. Utev, Variational inequalities with examples and an application to the Central Limit Theorem, Ann. Probab., 22 (1994), 1607-1618.

[4] M. Majsnerowska, A note on Poisson approximation by $w$-functions, Appl. Math., 25 (1998), 387-392. 